numeracy at year 5. This could be due to improved T1D management in South Australia

\section{RF02 EXPLAINING THE FALL IN CORONARY HEART DISEASE MORTALITY IN THE REPUBLIC OF IRELAND BETWEEN 2000 AND 2015 - AN IMPACT MODELLING STUDY}

'V Marasigan*, 'I Perry, ${ }^{1} \mathrm{~K}$ Balanda, ${ }^{2} \mathrm{~K}$ Bennett, ${ }^{1} \mathrm{Z}$ Kabir. ' $S$ Shool of Public Health, University College Cork, Cork, Ireland; ' ${ }^{2}$ Division of Population Health Sciences, RCSI, Dublin, Ireland

\subsection{6/jech-2019-SSMabstracts. 117}

Aim To investigate the proportional contributions of coronary heart disease (CHD) determinants to the observed CHD mortality rates in Ireland between 2000-2015.

Methods The validated IMPACT model on CHD mortality, which has been developed with the purpose of merging epidemiological data that is available for each country, was utilized for the estimations. Data on population statistics, CHD patient numbers, treatment uptakes and population trends on key risk factors (eg. smoking, total cholesterol, hypertension, obesity, DM and physical inactivity) were sourced from national registries, hospital administration systems, national health surveys, large cohort studies, international registries and meta-analyses. CHD Deaths Prevented or Postponed (DPPs) were used as outcome measurement.

Results CHD mortality in Ireland fell by 56\% (4060 fewer deaths), faster in women than in men (63\% vs 53\%), in the period 2000-2015 in those aged 25-84 years. Improvements in CHD risk factors, ie decrease in smoking prevalence $(5 \%)$, population systolic blood pressure $(-25 \%)$ and mean cholesterol serum levels $(-11 \%)$, contributed to $30 \%$ of the decline with 785 DPPs in men vs 425 in women. In women, both systolic blood pressure reductions and cholesterol reductions contributed equally (200 DPPs each), and decreased smoking prevalence contributed to 80 DPPs. Likewise, DPPs in men followed a similar trend (SBP - 825; total cholesterol- 250; and, smoking-110).

Improvements in cardiological treatments, specially in secondary prevention and heart failure treatments, contributed to approximately $60 \%$ of the observed decline (1620 DPPs in men and 825 in women). Both males and females benefitted the most DPPs from improvements in secondary prevention (850 and 355 DPPs, respectively). These gains were offset by increases in physical inactivity $(2 \%)$, diabetes prevalence $(6 \%)$ and BMI (4\%). Overall, improvements in CHD treatments were more beneficial to men whilst better risk factor contribution were higher in women. Advancements in CHD treatments were more beneficial than risk factors in all age groups. These proportions remained relatively consistent across a wide range of assumptions and estimates in a sensitivity analysis except for physical inactivity which has transcended the null line.

Conclusion The CHD mortality decline has continued between 2000-2015 of which $90 \%$ can be explained by improvements in cardiological treatments and population risk factors with the IMPACT modelling study. However, worsening trends in diabetes prevalence, obesity and physical inactivity have reversed the gains. Investments in improving $\mathrm{CHD}$ death determinants and targeted policies are necessary to sustain a further decline in CHD mortality rates in Ireland.

\section{RF03 \\ ESTIMATING THE OCCURRENCE OF DIABETES AT THE END OF LIFE USING MULTIPLE CAUSE OF DEATH DATA LINKED WITH PRIMARY CARE, HOSPITAL CARE AND MEDICATION PRESCRIPTION DATA}

${ }^{1} \mathrm{M}$ Mitratza*, ${ }^{1} \mathrm{AE}$ Kunst, ${ }^{2} \mathrm{P}$ Harteloh, ${ }^{3} \mathrm{MMJ}$ Nielen, ${ }^{1,2}{ }^{2} \mathrm{~B}$ Klijs. ${ }^{1}$ Department of Public Health, Academic Medical Centre, University of Amsterdam, Amsterdam, The Netherlands; ${ }^{2}$ Department of Health and Care, Statistics Netherlands, The Haque, The Netherlands; ${ }^{3}$ Netherlands Institute for Health Services Research (NIVEL), Utrecht, The Netherlands

\subsection{6/jech-2019-SSMabstracts.118}

Background Cause-of-death statistics underestimate the end-oflife occurrence of many diseases, including diabetes. Our aim is to estimate the end-of-life occurrence of diabetes by combining multiple cause of death data with register data covering primary care, hospital care and medication prescriptions. We investigate the added value of each medical register and assess the extent to which reporting of diabetes as a cause of death is associated with disease severity.

Methods Our study population consisted of all deaths in the Netherlands (2015-2016) included in the Dutch primary care database (NIVEL-PCD; $\mathrm{N}=18.162$ ). The proportion of deaths with diabetes (type I or II) within the last two years of life was calculated using cause of death and medical register data in isolation and combined. We assessed whether the proportion of diabetes reported in the causes of death registries varied according to disease severity as defined by medication prescriptions.

Results Of all deaths, $2.2 \%$ had diabetes reported as the underlying cause of death, while $7.7 \%$ of the death certificates mentioned diabetes. Primary care registration yielded the highest rate in isolation (27.1\%), followed by the medication $(22.4 \%)$ and the hospital-any diagnosis (17.1\%) data, while hospital-main diagnosis was limited (1.1\%). According to all data sources combined, $28.7 \%$ of the study population had diabetes at the end of life. Of all deaths among those who were prescribed insulin (indicating severe diabetes), 11.9\% had diabetes recorded as the underlying cause of death and $35.8 \%$ of the death certificates mentioned diabetes as a cause of death. For patients using oral antidiabetic medication, these proportions were only $5.7 \%$ and $24.2 \%$, and for patients not using antidiabetic medication $4.2 \%$ and $16.7 \%$.

Conclusion More than one fourth of the Dutch population has diabetes at the end of life. Only a minority of this endof-life diabetes occurrence is recorded as a cause of death, even for persons with severe diabetes. In the Netherlands, combining primary care data with multiple causes of death allows to find most cases with diabetes at the end of life.

\section{RF04 RISK OF BREAST CANCER AND OCCUPATIONAL EXPOSURE TO ORGANIC SOLVENTS: RESULTS OF THE CECILE STUDY, A POPULATION-BASED CASE-CONTROL STUDY IN FRANCE}

${ }^{1} \mathrm{~L}$ Radoï, ${ }^{1} \mathrm{E}$ Cordina-Duverger*, ${ }^{2,3} \mathrm{C}$ Piloget, ${ }^{1,2} \mathrm{P}$ Guénel. ${ }^{1}$ Center for Research in Epidemiology and Population Health (CESP), Inserm UMR 1018, University Paris-Sud, University Paris-Saclay, Villejuif, France; ${ }^{2}$ Occupational Health Department, Santé Publique France, National Agency for Public Health, Saint-Maurice, France; ${ }^{3}$ Epidemiological research and surveillance unit in transport, occupation and environment, Claude Bernard Lyon University, Lyon, France

\subsection{6/jech-2019-SSMabstracts. 119}

Background Breast cancer is the leading cause of cancer death in women worldwide. Besides reproductive and hormonal 